\title{
Modified Dachaihu Decoction Regulates FOXO3a Acetylation Activated Autophagy and Relieving Insulin Resistance in Obesity
}

\author{
Cong Long, Yang Gao, Xuke Han, Suwen Li and Qiu Chen* \\ Hospital of Chengdu University of Traditional Chinese Medicine, Chengdu 610072, Sichuan, China \\ Received: November 20, 2021; Accepted: November 26, 2021; Published: December 07, 2021
}

${ }^{\star}$ Corresponding author: Qiu Chen, Hospital of Chengdu University of Traditional Chinese Medicine, Chengdu 610072, Sichuan, China; Email: chenqiu1005@cdutcm.edu.cn

\begin{abstract}
Background

The previous studies of our research group indicate that the weakening of mitochondrial autophagy function is the key mechanism of obesityinduced insulin resistance, and Mitochondrial autophagy mediated by PINK1/Parkin pathway can reverse mitochondrial dysfunction. Recently, we found that FOXO3a, as an upstream regulator of PINK1, has been found to play a key role in regulating mitochondrial autophagy.However,FOXO3a is regulated by deacetylation.
\end{abstract}

\section{Objective}

To explore whether Modified Dachaihu Decoction can regulate liver mitochondrial autophagy mediated by the PINK1/Parkin signal pathway by regulating the expression of FOXO3a acetylation.

\section{Methods}

Establish cell models. They were divided into three groups (blank control group, model control group, and Modified Dachaihu Decoction group). The supernatant was extracted and determined by a biochemical method; The insulin sensitivity of each group was evaluated by a $3 \mathrm{H}-\mathrm{D}$-glucose incorporation test; MDA and TNFa IL-6 in the supernatant were detected by ELISA level; The level of SOD was detected by spectrophotometry.The expression of mitochondrial autophagy-related proteins and the expression of FOXO3a and ace-FOXO3a were measured by Western blot.

\section{Results}

Compared with the model control group, the Modified Dachaihu Decoction group increased insulin sensitivity, and The levels of TNF- $\alpha$ IL-6, and MDA decreased, while the activity of SOD increased $(\mathrm{P}<0.05)$. Western blot showed that compared with the model control group, the expression of mitochondrial autophagy-related proteins and FOXO3a in the Modified Dachaihu Decoction group increased, and the expression of ace-FOXO3a decreased $(\mathrm{P}<0.05)$.

\section{Conclusions}

We speculate that in this experiment, Modified Dachaihu Decoction may regulate mitochondrial autophagy mediated by PINK1/ Parkin signal pathway by downregulating the expression of FOXO3a acetylation, to reduce Hepatic Insulin Resistance in Obesity.

Keywords: FOXO3a Acetylation, Autophagy, Hepatic Insulin Resistance 